\title{
Theoretical and Experimental Investigations on Solar Photovoltaic Driven Thermoelectric Cooler System for Cold Storage Application
}

\author{
S. C. Kaushik, Ranjana Hans, and S. Manikandan
}

\begin{abstract}
This paper theoretically analyses and experimentally investigates the performance of Solar Photovoltaic driven thermoelectric cooler system for cold storage application. Small cold storage box of 3 liter capacity has been used for this investigation. The experimental setup has been made and analyzed for its performance in the composite climate of Delhi, India. Experimental results demonstrated that this unit could maintain the temperature of 10-15 degree Celsius inside the cold box. Moreover, energy and exergy analysis of the PV-TEC system has been performed to identify and quantify the irreversibilities occurring in the system. The performance of the system depends on incoming solar insolation and the temperature difference between the hot and cold sides for thermoelectric cooler module. It is expected that the cooler have potential for cold storage of food, vaccine and milk products in remote areas where electric power supply is not available.
\end{abstract}

Index Terms-Solar, thermoelectric cooler, cold storage.

\section{INTRODUCTION}

The most significant application of refrigeration is in food preservation, weather it is by way of processing or for cold storage. The preservation of food is defined as the preservation of palatability and nutritive value of food preventing the natural spoilage with respect to time. Solar refrigeration is thought as one of the best techniques to address this issue, due to its good match to the variation of solar radiation; namely, the supply of sunshine, and the cooling output of a solar refrigeration system reach maximum levels at the same season. The thermoelectric refrigeration system (TEC), which has the merits of being light, reliable, noiseless, rugged, and low cost in mass production, uses electrons rather than refrigerant as a heat carrier, and is feasible for outdoor purposes in cooperation with solar photovoltaic (PV) cells, in spite of the fact that its coefficient of performance is not as high as for a vapor compression cycle [1]. The theory of combined solar thermoelectric refrigerator is proposed and for its optimum operation, the ratio of number of thermocouples required is given by Vella et al. [2]. After that, a small prototype of thermoelectric refrigerator powered by solar photovoltaic solar collectors was proposed by Sofrata [3]. A theoretical model of thermoelectric device has been

Manuscript received July 24, 2015; revised September 18, 2015.

S. C. Kaushik is with Centre for Energy Studies, Indian Institute of Technology Delhi, Hauz Khas, Delhi-110016, India (e-mail: kaushik@ces.iitd.ac.in).

S. Manikandan and Ranjana Hans are with the Centre for Energy Studies, Indian Institute of Technology Delhi, Hauz Khas, Delhi-110016, India (e-mail: manikandan@ces.iitd.ac.in,ranjana1219@rediffmail.com). projected for geometric analysis and performance prediction of the performance in power generation mode [4]. A complete survey of solar driven thermoelectric technologies and their applications in various areas is also presented [5]. An investigation of optimal performance of solar-driven thermoelectric generator for optimization of efficiency is performed by Chen [6]. Afterwards, four different possible configurations of hybrid Solar-thermoelectric generator were considered and efficiency in different modes has been presented [7]. A hybrid design of generation system consisting of solar cells, thermoelectric generator and a heat collector producing $393 \mathrm{~mW}$ power is presented by Deng et al. [8]. Dia et al. [9] investigated solar driven thermoelectric refrigerator which has many advantages of light weight, noiseless and rugged performance, no hazardous refrigerants used and low cost in mass production. Kaushik and Manikandan [10] evaluated the energy and exergy analysis of the annular thermoelectric generator system and found that the energy efficiency is less than the exergy efficiency and exergy efficiency is the true measure of the efficiency to be addressed in any thermodynamic system. Various authors [11]-[14] have analyzed the thermoelectric cooler operated by thermoelectric generator without maximum power point tracking, with maximum power point tracking and with solar thermoelectric generator.

In this paper, the authors have fabricated a small prototype of solar photovoltaic driven thermoelectric refrigerator for cold storage of food and medicines and carried out its experimental investigation for the analysis of system performance. This design is compact, easy to operate and beneficial for remote rural areas where enough electricity supply is not there.

\section{Methodology}

\section{A. Solar Cooling Options}

The conventional refrigeration and space conditioning devices are based on vapour compression machines and need electrical energy and/or fossil fuels for their operation. With the advent energy crisis and the realization the depleting nature of the fossil fuels, the search for the use of alternative energy sources become an essentiality. Solar energy has a very high potential amongst renewable sources of energy since it is abundantly available and is an inexhaustible global source of energy having no pollution hazards associated with it [15].

Solar cooling is one of the most important aspects of solar 
energy conversion system, and it is more attractive because of the following factors:

- The demand for cooling is generally the greatest at times of maximum availability of solar radiation

- The cooling is far more needed in hotter regions than in colder climes.

Solar energy operated refrigeration, air conditioning and cold storage systems have great demand in tropical countries. Due to shortage of electric power in rural parts of the country there is acute shortage of cold storage facilities, as a result of which thousands of tonnes of perishable materials are being wasted every year.

The solar cooling options includes solar vapour absorption systems, solar vapour adsorption systems, solar photovoltaic-vapour compression systems and solar thermoelectric systems etc. In the above mentioned systems solar photovoltaic-vapour compression systems has been commercialized and widely used for cold storage and vaccine preservation in remote rural areas.

The disadvantages of solar photovoltaic-vapour compression systems are, it uses refrigerant compounds such as chloro fluro carbons (CFCs). Therefore, it contribute to the global warming and ozone depletion. Moreover, it have moving parts (compressor and fans) so that it require regular maintenance and this kind of system should be installed in such a way that its position should be unaltered. All these problems can be solved if the vapour compression system has been replaced by thermoelectric cooler.

\section{B. Solar Thermoelectric Cooler}

Solar thermoelectric cooling system is a direct energy conversion system for converting solar energy into electricity and utilize that electric power output to the cool the space. The cooling power output and the energy efficiency of the thermoelectric cooling system is less than the conventional vapour compression refrigeration system for higher power levels, but the efficiency of the thermoelectric cooling system is higher at lower power levels. The efficiency of vapour compression refrigeration system is low at lower power levels and its structural limitations will not allow VCR systems apt for lower cooling power levels like electronic chip cooling, small ice box cooling (0.5l), vaccine box cooling etc. Therefore, for such low cooling power applications thermoelectric cooling will be best suited.

The advantages of thermoelectric cooling includes low maintenance cost, transportable, low space requirement, noiseless operation and it can be installed in any orientation etc.

A prototype of a solar photovoltaic driven thermoelectric cooler have array of solar cells, DC/DC converter, storage battery and thermoelectric cooler module. The block diagrammatic representation of solar PV thermoelectric cooling system is shown in Fig. 1. During daytime the solar cells receive radiant solar energy from the sun and convert it into electrical energy, which is supplied to the thermoelectric cooler module installed inside the storage box. If the amount of electric power produced by the solar PV is sufficient to meet the cooling demand of the storage box, then the surplus electric power can be stored in the battery. If the solar power is deficit to meet the cooling load, the battery will supply power to the thermoelectric cooler. If the solar cells cannot produce sufficient electric power e.g., on cloudy or rainy days the storage battery will act as a backup power source. The DC/DC controller plays a role in maintaining constant voltage to the thermoelectric cooler from the solar PV panel. During night, the storage battery is used to power the thermoelectric cooler.

The cold side of the module is placed inside the cooler and the hot side is set outside. A fin-type heat exchanger is attached with the hot side of the thermoelectric cooler. In designing a portable thermoelectric cooler driven by solar cells, weight and cost of the total system are of great importance. It is expected that the system would be as light as possible and would not be expensive. For the thermoelectric refrigerator described above, a thermoelectric cooling module and electric power supply are extremely necessary. Since the storage battery is the heaviest component and the solar cells are most expensive. In this paper, the experiment work is focused mainly on testing the performance of the solar driven thermoelectric cooler during daytime without the need of a storage battery, and therefore, battery is not involved in the discussion of material characteristics of a thermoelectric module.

\section{Theoretical Basis and Experimental Setup}

The block diagram of the experimental setup of solar PV thermoelectric cooler is shown in Fig. 1. Solar photovoltaic panel is used to supply input power to the thermoelectric refrigerator through a DC/DC convertor. The power output from the Solar PV panel can be evaluated as

$$
P_{\text {Solar }}=S A_{P V} \eta_{P V}
$$

whereas, $S$ is the solar insolation rate, $A_{P V}$ is the area of solar panel and $\eta_{P V}$ is the energy conversion efficiency of solar power to electrical power.

The thin film solar panel of Sharp-NE78T1 $\left(78 \mathrm{~W}_{\mathrm{p}}\right)$ is chosen to drive 50W Peltier module with power backup of $12 \mathrm{~V}$ battery. A buck type DC/DC convertor has been employed to supply input voltage of $12-12.4 \mathrm{~V}$ to the thermoelectric cooling module. The experimental readings has been taken for a typical sunny day from 9:00am - 6:00pm and the results obtained from this study are graphically presented in this paper. The solar insolation rate of the location is measured parameters by using a Pyranometer (The Eppley precision spectral model PSP36851F3 by Eppley Laboratory Inc.), input voltage, current to the thermoelectric cooling module, ambient temperature, temperature of hot side and cold side of the thermoelectric cooler and the temperature of refrigerated object were measured to evaluate the performance of the solar PV thermoelectric cooling system.

The heat infiltration from the environment to the cold space of the cooler due to conduction, convection and radiation is neglected. The other parameters are calculated using the following equations (1)-(6)

The electrical current required for thermoelectric cooler is given as

$$
I=\left(V-\alpha\left(T_{h}-T_{c}\right)\right) / R
$$

where, $\alpha$ is the See beck coefficient of a thermoelectric 
cooling module, it has the unit of $(\mathrm{V} / \mathrm{K})$.

The electrical input power supplied to the thermoelectric cooler is the electrical power output from the solar PV panel

$$
P_{i n}=P_{\text {Solar }}=\alpha I\left(T_{h}-T_{c}\right)+I^{2} R
$$

The cooling power of the thermoelectric cooler can be given as

$$
Q_{C}=\alpha I T_{c}-I^{2} R / 2-K_{t}\left(T_{h}-T_{c}\right)
$$

where, $R$ is the electrical resistance of the thermoelectric module. $T_{h}$ and $T_{\mathrm{c}}$ are the temperatures of hot side and cold side respectively, where $K_{t}$ is the total thermal conductance of the thermoelectric cooling module.

The Figure of Merit of the thermoelectric module can be calculated as

$$
Z=\alpha^{2} / R K_{t}
$$

The maximum temperature difference which can be obtained in a thermoelectric cooler is estimated as

$$
\Delta T_{\max }=Z T_{c}^{2} / 2
$$

The maximum energy efficiency of the thermoelectric cooling system is given as

$$
C O P=\left(\left(T_{c} /\left(T_{h}-T_{c}\right)\right)\left(M_{c}-T_{h} / T_{c}\right)\right) /\left(M_{c}+1\right)
$$

where,

$$
T_{M}=\left(T_{h}+T_{c}\right) / 2 \text { and } M_{c}=\sqrt[2]{Z T_{M}+1}
$$

The maximum cooling capacity is defined as

$$
Q_{C \max }=K_{t}\left(\Delta T_{\max }-\Delta T\right)
$$

The power output of the solar panel can also be written as

$$
P_{\text {Solar }}=V_{O C} I_{S C} F F=V_{m p} I_{m p}
$$

where $V_{\mathrm{oc}}$ is the open circuit voltage, $I_{\mathrm{SC}}$ is the short circuit current, $F F$ is the fill factor and $V_{\mathrm{mp}}, I_{\mathrm{mp}}$ are the maximum voltage and current of solar photovoltaic panel which can be obtained.

The efficiency of the solar panel is given as

$$
\eta_{P V}=\left(V_{O C} I_{S C} F F\right) / A_{P V} S
$$

The efficiency of solar photovoltaic system depends on open circuit voltage, short circuit current, fill factor, area of panel and solar insolation rate.

Then the combined system efficiency of the solar PV thermoelectric cooling system can be evaluated as

$$
C O P=Q_{C} / P_{\text {Solar }}
$$

The combined system exergy efficiency of the solar PV thermoelectric cooling system can be evaluated as

$$
\Psi=\left(E_{Q c} / P_{\text {Solar }}\right)=1-\left(\text { Irreversibilities } / P_{\text {Solar }}\right)
$$

where, $E_{Q c}$ is the exergy of the cooling power, which can be obtained by multiplying Carnot factor $\left(T_{c} / T_{o}\right)-1$ with $Q_{c}$ and $T_{o}$ is the environment temperature.

Exergy analysis is useful to identify and quantify the recoverable and irrecoverable (irreversibilities) exergy losses occurring in any thermodynamic system, so that the actual corrective measures can be taken to improve the performance of the system.

The exergy analysis in a solar thermoelectric system is necessary because, it can identify and quantify the internal and external irreversibilities based on the factors which causes those irreversibilities. Therefore, proper corrective measures can be taken to improve the true performance of the thermoelectric system.

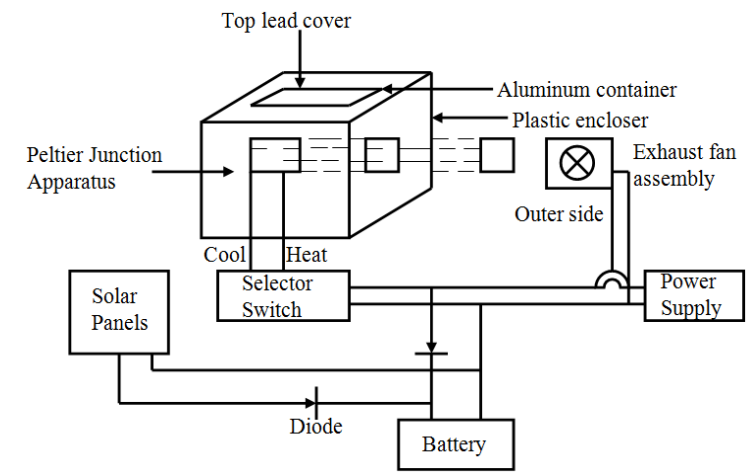

Fig. 1. Block diagram of Solar PV driven thermoelectric refrigerator system.

\section{RESUlT AND DISCUSSIONS}

\section{A. Theoretical Analysis}

The solar PV thermoelectric cooler have been theoretically analyzed in MATLAB Simulink environment for different cold side temperature $\left(T_{c}\right)$ with constant hot side temperature $\left(T_{h}\right)$ for varying solar radiation. The hot side temperature is fixed at $303 \mathrm{~K}$ and the cold side temperature is varied from $273 \mathrm{~K}$ to calculate the cooling power output, energy efficiency, exergy efficiency and irreversibilities in the solar PV thermoelectric cooling system. The solar radiation is assumed to be varying from $200 \mathrm{~W} / \mathrm{m}^{2}$ to $850 \mathrm{~W} / \mathrm{m}^{2}$ for a normal day. The single crystalline solar cell PV panel is used for the theoretical analysis, which has energy conversion efficiency of $14 \%$. The power rating of PV panel is $168 \mathrm{~W}_{\mathrm{p}}$. A DC/DC converter is used which supplies input to the thermoelectric cooler at constant voltage of $12 \mathrm{~V}$.

The variation in cooling power of the solar PV thermoelectric cooler for the time of a day is shown in Fig. 2. It shows that the cooling power decreases with increase in temperature difference between hot and cold junction of thermoelectric cooler $(\Delta T c)$. The other point should be noted from this figure is that the cooling power of the system is higher at mid of the day, i.e., when the cooling power requirement is more since the atmospheric temperature is more. This is one of the important features of the solar 
powered refrigeration systems.

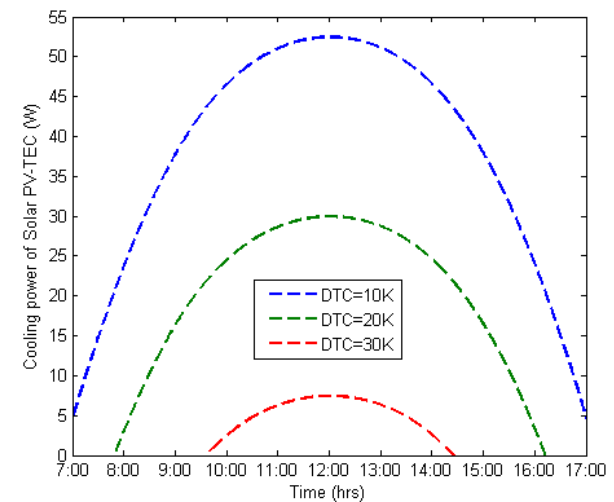

Fig. 2. Cooling power of solar PV-Tec system with time of the day.

The variation in energy efficiency of the solar PV thermoelectric cooler with time of a day is shown in Fig. 3. It shows that the energy efficiency of the solar PV thermoelectric cooler decreases with increase in $\Delta T c$, because the cooling power decreases with increase in $\Delta T c$. Moreover, it can be seen that the energy efficiency of the system decreases during the mid of the day because the power input to the thermoelectric cooler $\left(P_{\text {Solar }}\right)$ increases the cooling power of thermoelectric cooler, thereby decreases its energy efficiency.

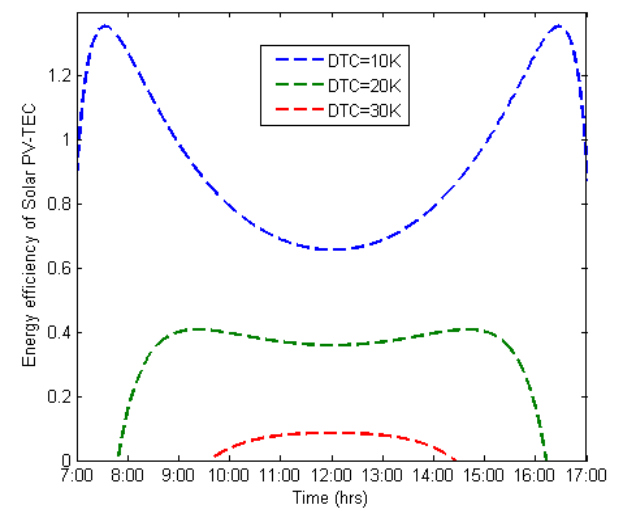

Fig. 3. Energy efficiency of solar PV-TEC system with time of the day.

The variation in energy efficiency and the cooling power of the solar PV thermoelectric cooling system for different solar radiation is shown in Fig. 4. It shows that the cooling power increases with radiation and the energy efficiency decreases with increase in solar radiation.

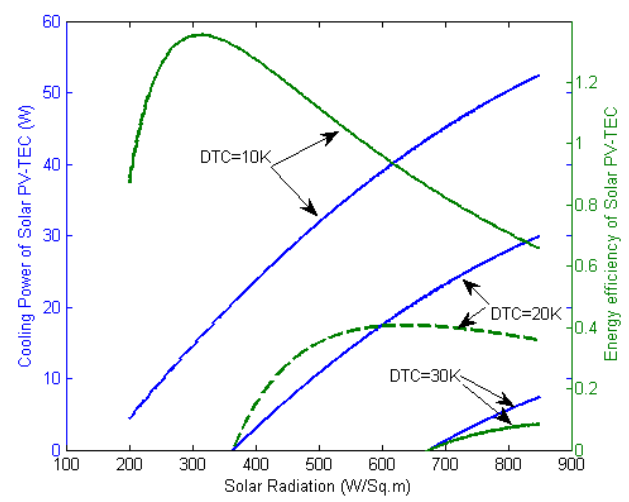

Fig. 4. Effect of solar radiation on the energy efficiency and cooling power of solar PV-TEC system.

Fig. 5 shows the variation of irreversibilities in Solar
PV-TEC system with time of the day. It shows that the irreversibilities in the system initially increases with time of the day and are maximum at mid noon. As the temperature difference between hot and cold side increases, irreversibilities in the system also goes up.

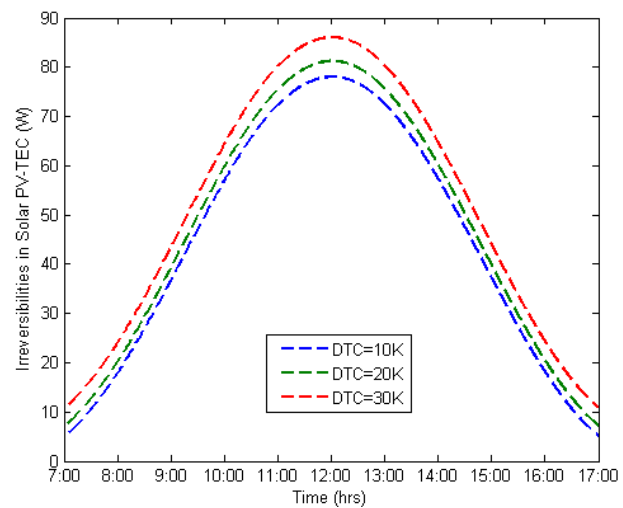

Fig. 5. Irreversibilities in solar PV-TEC system with time of the day.

The variation in exergy efficiency of the PV-TEC system for different $\Delta T c$ is presented in Fig. 6. It shows that the exergy efficiency follows the similar pattern of energy efficiency, because as the solar radiation increases, the power output of the solar PV increases, thus decreasing the exergy efficiency and increasing the cooling power. The exergy efficiency of the PV-TEC system is lower than the energy efficiency because the exergy output of the thermoelectric cooler is low.

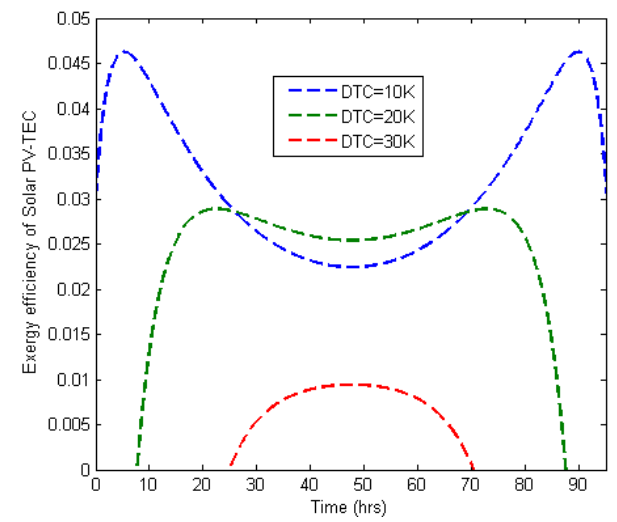

Fig. 6. Exergy efficiency of solar PV-TEC system with time of the day

\section{B. Experimental Setup}

The experimental setup of the solar thermoelectric cooling system is shown in Fig. 7. The experiment has been carried out at Sohna, Haryana located at $28.25^{\circ} \mathrm{N}-77.07^{\circ} \mathrm{E}$ in the composite climatic zone of India for a normal sunny day.

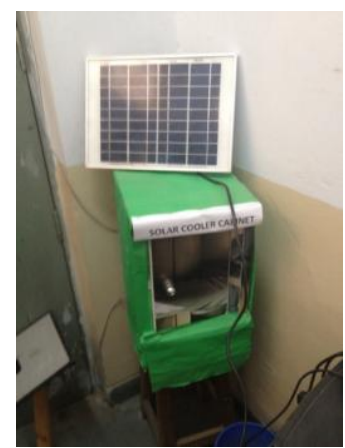

Fig. 7. Experimental setup of solar PV thermoelectric cooler system. 
The thermoelectric cooling module is connected with the power supply (i.e., the solar panel). The thermoelectric cooling module is installed in such a way that, its cold side is facing inside the cooler box and the hot side is facing outside (i.e., environment). The temperatures of the hot and cold sides of the thermoelectric module are measured by thermocouple wires connected to the hot and cold sides of the thermoelectric cooling module. The hot side of the thermoelectric cooling module is attached to the heat sink whereas the cold side of the thermoelectric module is used to cool the cooler cabinet. The heat sink is attached to the hot side of the thermoelectric module to reduce the thermal resistance so as to transfer the heat more efficiently to the atmosphere. The heat sink is connected with the cooling fan that is used to further ease the heat transfer process Fig. 8 shows the pyranometer that has been used to measure solar insolation rate.

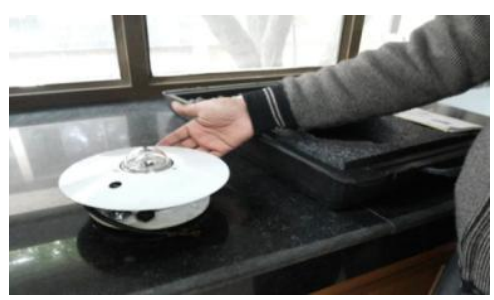

Fig. 8. Pyranometer - An instrument that measures solar insolation rate.

Fig. 9 shows the variation of cold side temperature of the thermoelectric cooler and ambient temperature with loaded and unloaded condition on a typical sunny day of $3^{\text {rd }}$ May, 2014. The unloaded condition means that the cooling cabinet is empty and load condition means that $500 \mathrm{ml}$ of water is used as the load. It can be seen from Fig. 7 that the temperature of thermoelectric module decreases from $23^{\circ} \mathrm{C}$ to $13^{\circ} \mathrm{C}$ as input solar insolation increases for unloaded condition and the temperature decreases from $23^{\circ} \mathrm{C}$ to $16^{\circ} \mathrm{C}$ with loaded condition.

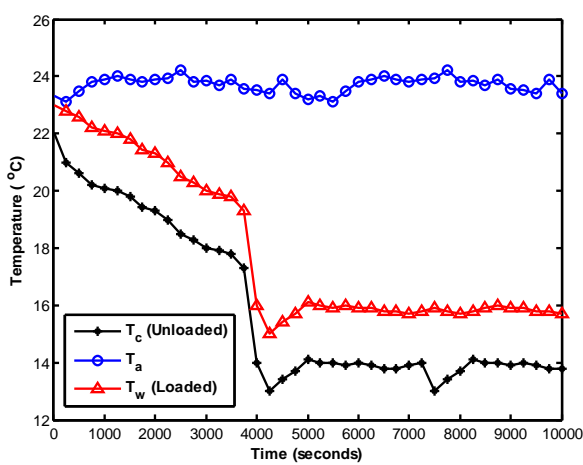

Fig. 9. Variation of cold side temperature and ambient temperature with loaded and unloaded condition.

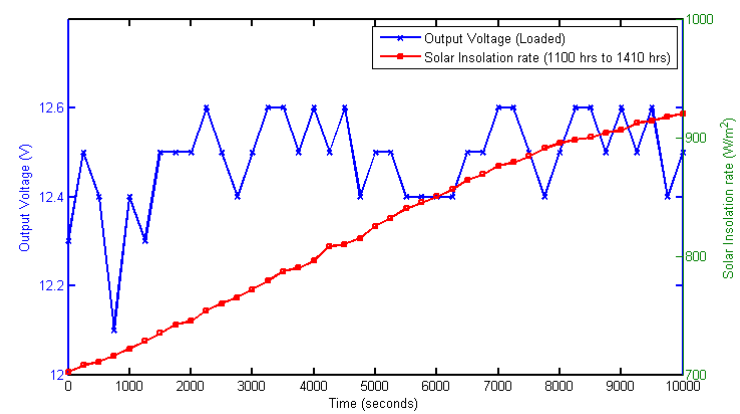

Fig. 10. Variation of solar radiation and input voltage to the thermoelectric cooler with time.
The variation in solar insolation rate and voltage input to the thermoelectric module for the time of a day is shown in Fig. 10. It shows that the voltage input to the thermoelectric cooler is maintained almost constant throughout the day with the help of DC/DC converter. Therefore, it can be concluded that the power input to the thermoelectric cooler is increased over the time, therefore, it increases the cooling power, so the cold space temperature drops.

It can be seen from this study that the solar thermoelectric cooler system can maintain $13^{\circ} \mathrm{C}-16^{\circ} \mathrm{C}$ at its cold space under loaded condition.

It can also be seen that the system is compact and transportable, and can be used for storing food, drinks, and vaccines for remote rural areas where grid supply is not accessible.

\section{CONCLUSIONS}

The theoretical analysis and the experimental investigations on solar thermoelectric cooler system have been carried out. The following conclusions can be drawn from this study:

- The solar PV thermoelectric cooling system can maintain 13-16 degree Celsius temperature with $500 \mathrm{ml}$ of water as cooling load.

- This system can reach the temperature of 15 degree Celsius within 15 minutes.

- The theoretical analysis shows that the cooling power increases with solar radiation and the energy efficiency decreases with solar radiation.

- It is also clear that the cooling power is high during the midday, when the cooling power requirement is high.

This solar PV thermoelectric cooling system can be best suited for vaccine cooling, foods and milk products in remote rural areas.

It can be seen from the theoretical analysis and experimental results that the cooling power, energy efficiency and exergy efficiency are low for this system, but if the thermoelectric materials with high figure of merit is found, then these systems will gain practical importance, and it will become true in near future.

\section{REFERENCES}

[1] S. A. Omer and D. G. Infield, "Design optimization of thermoelectric devices for solar power generation," Solar Energy Materials and Solar Cells, vol. 53, pp. 67-82, 1998 .

[2] G. J. Vella, L. B. Harris, and H. J. Goldsmid, "A solar thermoelectric refrigerator," Solar Energy, vol. 18, pp. 355-359, 1976

[3] H. Sofreta, "A thermoelectric refrigerator powered by photovoltaic solar collectors," Applied Energy, vol. 18, pp. 137-142, 1984

[4] H.-X. Xi, L.-G. Luo, and G. Fraisse, "Development and applications of solar based thermoelectric technologies," Renewable and Sustainable Energy Reviews, vol. 11, pp. 923-936, 2007.

[5] D. S. Kim and C. A. I. Ferreira, "Solar refrigeration options - A state-of-the-art review," International Journal of refrigeration, vol. 31, pp. 3-15, 2008.

[6] J.-C. Chen, "Thermodynamic analysis of a solar driven thermoelectric generator," Journal of Applied Physiscs, vol. 79, pp. 2717-2721, 1996

[7] E. A. Chavez-Urbiola, Y. V. Vorobiev, and L. P. Bulat, "Solar hybrid system with thermoelectric generators," Solar Energy, vol. 86, pp. 369-378, 2012.

[8] Y. Deng, W. Zhu, Y. Wang, and Y. Shi, "Enhance performance of solar driven photovoltaic thermoelectric hybrid system in an integrated design," Solar Energy, vol. 88, pp. 182-191, 2013. 
[9] Y. J. Dai, R. Z. Wang, and L. Ni, "Experimental investigation and analysis on a thermoelectric refrigerator driven by solar cells," Solar Energy Materials and Solar Cells, vol. 77, pp. 377-391, 2003.

[10] S. Kaushik and S. Manikandan, "The influence of Thomson effect in the energy and exergy efficiency of an annular thermoelectric generator," Energy Conversion and Management, vol. 103, pp. 200-207, 2015.

[11] G. J. Vella, L. B. Harris, and H. J. Goldsmid, "A solar thermoelectric refrigerator," Solar Energy, vol. 18, pp. 355-359, 1976.

[12] L.-G. Chen, F.-K. Meng, and F.-R. Sun, "Effect of heat transfer on the performance of thermoelectric generator-driven thermoelectric refrigerator system," Cryogenics, vol. 52, pp. 58-65, 2012.

[13] S. Manikandan and S. C. Kaushik. "Thermodynamic studies and maximum power point tracking in thermoelectric generator-thermoelectric cooler combined system," Cryogenics, vol. 67, pp. 52-62, 2015.

[14] N. M. Khattab and E. T. E. Shenawy, "Optimal operation of thermoelectric cooler driven by solar thermoelectric generator," Energy Conversion and Management, vol. 47, pp. 407-426, 2006

[15] S. C. Kaushik, Solar Refrigeration and Space conditioning, Jodpur: Divyajyoti Prakasan, 1989.

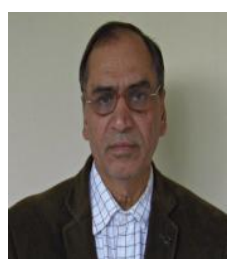

S. C. Kaushik is a professor in CES at IITDelhi. He received his Ph.D. in plasma science from IIT Delhi after his distinguished first position in master's degree in science (electronics) from Meerut University. His research fields of activities include thermal science and engineering; energy conservation and heat recovery, solar refrigeration and airconditioning, solar architecture, and thermal storage and power generation. He has made significant contributions in these fields as evident by his above 300 research publications in Journals of repute at national and international levels. He is a pioneer researcher on exergy analysis and finite time thermodynamics of energy systems at national and international level and a leading expert on alternative refrigeration and air conditioning technologies.

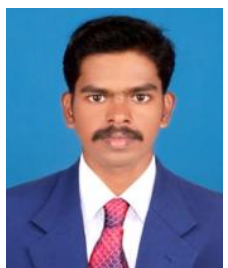

S. Manikandan has obtained his B.E (electrical engineering) from Anna University Chennai and M.E (energy engineering) from Anna University Tirunelveli and he is currently pursuing the $\mathrm{PhD}$ from Centre for Energy Studies (CES) at Indian Institute of Technology Delhi (IITD) on solar thermoelectric cooling and power generation systems.

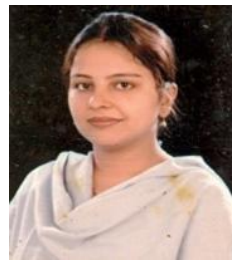

Ranjana Hans is an assistant professor in the Renewable Energy Department, Amity University, Manesar, Haryana, India and currently she is pursuing the $\mathrm{PhD}$ from Centre for Energy Studies (CES) at Indian Institute of Technology Delhi (IITD) on thermoelectric devices and power generation systems. 